Revue internationale P.M.E.

Économie et gestion de la petite et moyenne entreprise

\title{
Le financement de l'actif immatériel des nouvelles entreprises technologiques et innovantes
}

\section{Alain Marion}

Volume 8, numéro 3-4, 1995

URI : https://id.erudit.org/iderudit/1008365ar

DOI : https://doi.org/10.7202/1008365ar

Aller au sommaire du numéro

Éditeur(s)

Presses de l’Université du Québec

ISSN

0776-5436 (imprimé)

1918-9699 (numérique)

Découvrir la revue

Citer cet article

Marion, A. (1995). Le financement de l'actif immatériel des nouvelles entreprises technologiques et innovantes. Revue internationale P.M.E., 8(3-4), 205-231. https://doi.org/10.7202/1008365ar
Résumé de l'article

L'importance de l'effort d'investissement induit par la dynamique du processus de développement technologique génère dans les ETI (entreprises technologiques et innovantes), particulièrement celles de création récente ou de taille réduite, un besoin structurel de capital. Ce besoin, contrepartie de l'actif immatériel créé, est couvert, dans le cadre d'une hiérarchie de financement spécifique, d'une manière préférentielle par l'autofinancement, et pour le solde, par un recours privilégié aux capitaux propres, notamment au capital de risque.

Certains développements théoriques récents, notamment les travaux de Williamson sur l'adéquation des modes de financement au degré de spécificité des actifs, fournissent un cadre conceptuel original qui permet d'avoir une lecture explicative pertinente du comportement financier des ETI, complémentaire aux éclairages traditionnels de la théorie financière. Reste à approfondir la notion de spécificité appliquée aux actifs pour en renforcer le caractère opératoire.
Ce document est protégé par la loi sur le droit d'auteur. L'utilisation des services d’Érudit (y compris la reproduction) est assujettie à sa politique d'utilisation que vous pouvez consulter en ligne.

https://apropos.erudit.org/fr/usagers/politique-dutilisation/ 


\title{
Le financement de l'actif immatériel des nouvelles entreprises technologiques et innovantes
}

Alain MARION*

IAE de Lyon

\section{MOTS CLÉS}

Financement des PME - Actifs immatériels - Structure financière Actifs spécifiques - Hiérarchie des financements Développement technologique

\begin{abstract}
RÉSUMÉ
L'importance de l'effort d'investissement induit par la dynamique du processus de développement technologique génère dans les ETI (entreprises technologiques et innovantes), particulièrement celles de création récente ou de taille réduite, un besoin structurel de capital. Ce besoin, contrepartie de l'actif immatériel créé, est couvert, dans le cadre d'une hiérarchie de financement spécifique, d'une manière préférentielle par l'autofinancement, et pour le solde, par un recours privilégié aux capitaux propres, notamment au capital de risque.

Certains développements théoriques récents, notamment les travaux de Williamson sur l'adéquation des modes de financement au degré de spécificité des actifs, fournissent un cadre conceptuel original qui permet d'avoir une lecture explicative pertinente du comportement financier des ETI, complémentaire aux éclairages traditionnels de la théorie financière. Reste à approfondir la notion de spécificité appliquée aux actifs pour en renforcer le caractère opératoire.
\end{abstract}

\section{ABSTRACT}

The aim of this article is to study the capital structure choices in Small High Technology Firms. These firms are faced with a high rate of capital expenditure

* Professeur de sciences de gestion à l'Institut d'administration des entreprises (Université Lyon III). Directeur du Diplôme d'études supérieures spécialisées (DESS) de Diagnostic d'entreprise. Expert en Diagnostic d'entreprise auprès de la Cour d'appel de Lyon. Adresse : Institut d'administration des entreprises (IAE) de Lyon, Université Jean-Moulin Lyon III, 15, quai Claude-Bernard, B.P. 0638, 69239 Lyon, cedex 02, France. 
that generates structural needs of finance. This need, reflection of intangible asset created by accumulation of expenses, is covered preferably by internal finance, and for the remain, first by equity, secondly by debt.

Some recent theoritical developments, specially Williamson's works relative to the adequation between financial structures and characteristics of the assets, provides an original conceptual framework to explain financial behavior of Small High Technology Firms.

\section{RESUMEN}

El objeto del presente trabajo es el estudio de las decisiones tomadas en la estructura del capital de pequeñas empresas de alta tecnología (EAT). Dichas sociedades se enfrentan con tasas de gastos elevadas que generan necesidades estructurales de financiación. Estas necesidades, contrapartida del activo immaterial creado por la acumulación de gastos, se cubre principalmente gracias a un financiamiento interno, y en segundo lugar a travès de los fondos propios.

Algunas reflexiones teóricas recientes, especialmente los trabajos de Williamson relativos a la adecuación entre tipo de financiamiento y especificidades de las inversiones constituyen un marco conceptual original, complementario con los aportes tradicionales de la teoría financiera, que permite explicar el comportamiento financiero de las EAT. Queda pendiente un analisis más amplio del concepto de especificidad aplicado a las inversiones, que pueda mejorar su implementación.

\section{Introduction}

Des critères internationaux permettent désormais de définir et d'appréhender les entreprises technologiques et innovantes (ETI). Par entreprise technologique, nous entendons les entreprises qui développent ou exploitent des nouvelles technologies. Les entreprises innovantes sont celles qui lancent des produits ou des procédés nouveaux, ayant ou non un contenu technologique, à partir de technologies existantes.

L'OCDE caractérise les industries de haute technologie comme celles allouant plus de $4 \%$ de leur chiffre d'affaires aux dépenses de recherchedéveloppement (Lehoucq et Strauss, 1988). Par référence à cette approche, on peut considérer qu'une ETI est soit une entreprise appartenant à une des industries dites de haute technologie, soit une entreprise dont l'intensité technologique est supérieure à un seuil. La non-appartenance à un secteur de haute technologie n'interdit pas à une entreprise de revendiquer la qualité d'entreprise technologique ou innovante. Une récente enquête du ministère de l'Industrie montre que près des deux tiers des PMI françaises prévoyant poursuivre ou amplifier leur programme d'innovation appartiennent à des secteurs qui ne sont pas réputés être de haute technologie (Ministère de l'Industrie, 1993). 
L'étude du financement de l'actif immatériel des ETI s'avère un champ d'investigation particulièrement stimulant pour l'esprit, mettant en exergue quelques-unes des grandes questions de fond auxquelles la finance d'entreprise n'a pas encore apporté de réponses pertinentes (Brealey, Myers et Laroche, 1991). Pour s'en tenir à une liste réduite, citons notamment la nature de la relation entre les caractéristiques des actifs et le risque de défaillance financière, le problème de la détermination de la structure financière et, plus précisément, de la répartition entre capitaux propres et dette, et enfin le sujet de la séparabilité des décisions de financement et d'investissement. La liste est cependant loin d'être exhaustive, et nous aurons l'occasion dans le corps de l'article d'évoquer d'autres points restés à ce jour dans l'ombre de la théorie financière.

Il ne faudrait toutefois pas extrapoler exagérément à partir de ces quelques remarques introductives et conclure que le mutisme de la théorie financière est total quand il s'agit d'expliquer le comportement financier des ETI. Les développements récents de la théorie de l'agence consacrés à l'étude des relations contractuelles entre apporteurs de capitaux et dirigeants fournissent un cadre conceptuel particulièrement pertinent pour expliquer le rôle nécessaire que jouent les intermédiaires financiers, et notamment les sociétés de capitalrisque, dans le financement des ETI nouvelles ou de taille réduite (Mahieux, 1993). De même, les travaux de Williamson sur l'adaptation des modes de financement au degré de spécificité des actifs trouvent une application évidente dans le cadre de la problématique du financement de l'actif immatériel des ETI en fournissant une justification des modes de financement privilégiés par ces entreprises. Cependant, le poids, la nature et la spécificité des besoins financiers des ETI éclairent d'un jour particulier les problèmes non résolus de la finance d'entreprise.

Bien que la problématique présentée concerne l'ensemble des ETI, notre souci est d'analyser plus spécialement le comportement financier de la firme assumant un risque élevé et faisant face à une limitation dans l'accès aux financements externes. C'est pourquoi nous intégrons dans notre étude les caractéristiques des entreprises technologiques et innovantes de création récente, sachant que la taille s'avère un facteur fortement discriminant dans le comportement financier des entreprises alors que son influence sur le comportement innovateur a longtemps été sujet de controverses. Les avis concordent désormais pour considérer que la taille n'est pas un facteur discriminant du potentiel d'innovation des entreprises (Acs et Audrestsch, 1988). La création et le développement de nombreuses PME dans des secteurs à haute intensité technologique est là pour le montrer, remettant ainsi en cause l'avantage «théorique » attribué à la grande entreprise par de nombreux économistes qui s'appuyaient, pour défendre leur thèse, sur les économies d'échelle dont 
bénéficient les grandes entreprises et la réduction du risque permise par la diversification des activités. Gardons-nous cependant d'affirmer que les PME, grâce à la flexibilité que leur confère leur taille, font systématiquement preuve d'un dynamisme plus marqué en matière de comportement innovateur.

Généralement, la problématique du financement des ETI évoque les difficultés qu'éprouvent ces entreprises dans l'accès au capital de risque et aux aides à la recherche-développement. Difficultés particulièrement ressenties par les ETI de création récente auxquelles notre étude est consacrée. En effet, comme le souligne Geoffron (1991), «les PME innovatrices sont particulièrement vulnérables aux effets d'éviction des marchés de capitaux que subissent les PME ou à la sélection adverse qui affecte les entreprises présentant les projets les plus risqués».

Nous avons choisi d'étudier un aspect complémentaire: l'adaptation de la structure du capital aux caractéristiques spécifiques de l'actif. La première partie met en évidence les caractéristiques observées du comportement financier des nouvelles ETI, notamment l'importance qu'occupe l'immatériel au sein de l'actif, le profil atypique du risque selon les phases de développement technologique et, enfin, l'existence d'un besoin structurel de capitaux propres. La deuxième partie tente une lecture explicative du comportement financier des ETI à l'éclairage de la théorie financière, en s'appuyant notamment sur les travaux consacrés à l'adéquation des modes de financement au degré de spécificité des actifs.

\section{Les caractéristiques observées du comportement financier des nouvelles ETI}

La technologie et l'innovation sont consommatrices de capitaux. Le développement des ETI n'est assuré que dans la mesure où, préalablement, l'entreprise a procédé à l'accumulation d'un savoir-faire que l'application ultérieure à des occasions d'affaires commerciales permettra de valoriser sur le marché. C'est dans ce décalage temporel et dans son importance que se trouve l'explication d'une partie de la spécificité du modèle de développement des ETI. Procédant à une comparaison de l'entreprise traditionnelle ${ }^{1}$ et de l'ETI, Godener (1994) souligne que cette dernière est «dans une autre logique du fait de l'importance de sa recherche et développement: le savoir-faire de départ n'est pas suffisant et une période de mise au point du produit et du processus d'industrialisation constitue une phase à part entière de l'entreprise constituée».

1. Nous définissons l'entreprise traditionnelle comme étant celle qui fabrique des produits ou met en œuvre des procédés reposant sur l'utilisation de savoir-faire répandus dans le secteur. 
Cette caractéristique combinée à d'autres, notamment la difficulté des ETI de petite taille à maîtriser le risque au regard de la faible diversification de leur portefeuille d'activités, les handicaps dans la recherche de financements externes du fait de leur taille, explique les spécificités du comportement financier des nouvelles ETI, particulièrement le recours fréquent au capital de risque et l'accès privilégié aux aides publiques.

\subsection{Importance de l'actif immatériel dans les ETI}

Dans les ETI, l'actif immatériel repose principalement sur deux bases: d'une part, le capital technologique qui recouvre le stock de savoir et de savoir-faire accumulés par l'entreprise, d'autre part, le capital commercial qui traduit fondamentalement la durabilité du lien établi entre l'entreprise et ses clients.

En cela, les ETI ne sont pas différentes des PMI traditionnelles chez qui l'investissement en recherche et les investissements commerciaux représentent les deux tiers de l'effort d'investissement immatériel (Sofaris, 1988). La différence provient de l'intensité de ces dépenses beaucoup plus forte dans les ETI.

\subsubsection{Place de la recherche et développement et des dépenses de lancement dans la dynamique de croissance des ETI}

Nous avons défini les industries à haute densité technologique comme celles allouant plus de $4 \%$ de leur chiffre d'affaires à la recherche. En fait, l'intensité de recherche des entreprises appartenant à ces industries est sensiblement plus élevée et atteint $8 \%$ en moyenne (contre $2,7 \%$ pour l'ensemble de l'industrie et $0,3 \%$ pour les industries à faible densité technologique). Sachant que dans les industries de haute technologie, l'investissement matériel représente un peu plus de la moitié des dépenses de recherche (Lehoucq et Strauss, 1988), on constate que le total des investissements en matériel et en recherche représente près de $12 \%$ du chiffre d'affaires contre un montant inférieur à $5 \%$ dans les industries à faible densité technologique ${ }^{2}$. On est dans un rapport dépassant le simple au double qui ne saurait être, sur une longue période, sans incidence sur la structure financière.

Cependant, le taux de $12 \%$ n'est pas vraiment représentatif de l'ampleur des besoins totaux induits par l'effort d'investissement technologique. On considère généralement que les dépenses liées au lancement industriel et commercial consécutives à l'effort de recherche peuvent représenter jusqu'à 3 à 5 fois le coût de ces dernières (Larrera de Morel, 1992). Comme le soulignent

2. Ces données sont confirmées par l'enquête qu'a réalisée, en 1993, Isabelle Fau auprès d'un échantillon de 35 entreprises technologiques et innovantes implantées dans une pépinière de la région lyonnaise (Fau, 1993). 
Albert et Mougenot, dans les ETI, «l'investissement doit souvent être ponctuel et massif pour financer simultanément tous les aspects d'un projet global: mise au point technique, création d'un programme de vente, formation du personnel...» (Albert et Mougenot, 1988).

\subsubsection{Le processus de création de l'actif immatériel}

Le processus de création de l'actif technologique et commercial est l'aboutissement de l'option technologique développée par l'entreprise. Comme l'observe Larue de Tournemire (1991) «la logique des stratégies technologiques concerne aussi bien l'élaboration des technologies génériques que la constitution d'un potentiel technologique spécifique à la firme et valorisable dans de multiples couples produit-marché».

La création de l'actif immatériel résulte de l'accumulation dans le temps de dépenses qui, au regard de leur effet économique (amortissement sur une production future, développement de l'actif économique), méritent d'être qualifiées d'investissement.

La liste des investissements immatériels est étendue puisque y figurent les dépenses de recherche-développement, les dépenses de marketing (frais engagés pour la constitution d'un réseau, budgets alloués à la communication et à la promotion, etc.), les dépenses de formation, à tout le moins celles qui excèdent le seuil légal minimal, les dépenses de conception et de développement informatiques, enfin, les dépenses allouées à l'amélioration de la qualité des produits et des procédés.

L'hétérogénéité des dépenses ne doit cependant pas cacher le critère fédérateur qui permet de les réunir dans une catégorie économique homogène. En effet, toutes ces dépenses qui sont destinées à servir durablement l'activité de l'entreprise, et présentent par ailleurs une rémanence certaine qui traduit la durée de leur impact, contribuent à doter l'entreprise d'un avantage spécifique créateur de valeur.

Les investissements immatériels présentent certaines caractéristiques qui justifient d'autant plus leur regroupement. Ainsi, certains investissements immatériels, à la différence des investissements physiques, semblent bénéficier de rendements croissants. La raison semble en être que l'investissement immatériel, dont la nature des composantes est diverse, vient s'agréger au stock de compétences de l'entreprise dont il est susceptible, par son apport, de renforcer la valeur et l'efficience. L'investissement immatériel a un impact simultané sur plusieurs des leviers de performance de l'entreprise. En son temps, Hicks (1975) avait perçu l'existence de ce mécanisme de création de valeur en observant la synergie dégagée par la complémentarité des équipements. 
C'est certainement dans cette voie qu'est à rechercher l'explication de la supériorité des taux de rendement du capital technologique en comparaison de ceux du capital physique. De nombreuses études (Mairesse et Mohnen, 1990) montrent que l'investissement en recherche contribue à améliorer la productivité des entreprises. L'incidence positive ne s'arrête d'ailleurs pas aux seules entreprises qui en assument l'effort, mais s'étend aux autres entreprises du secteur. Comme le soulignent Mairesse et Mohnen, «même si les études ne sont que très imparfaitement comparables entre elles, on peut conclure que les taux de rendement de la recherche sont fortement positifs, pouvant aller jusqu'à $50 \%$, mais plus vraisemblablement de l'ordre de 15 à $25 \%$ ».

Une autre caractéristique des investissements immatériels repose sur la forme particulière de leur appropriation (Teillet, 1988), surtout quand cette dernière adopte la forme d'une incorporation humaine, comme dans le cas des dépenses allouées à la recherche et à la formation. Cette caractéristique induit une complexité évidente pour qui s'intéresse à la mesure de leur efficacité et pose, par ailleurs, la question de la pertinence de leur valorisation dans une optique patrimoniale (Marion, $1987 ; 1988 ; 1990$ ). Il peut y avoir en effet dissociation entre celui qui finance la création du capital immatériel et celui qui l'utilise. De là à considérer, comme le fait le Conseil national de l'information statistique (INSEE, 1993) que seules méritent la qualité d'investissement immatériel les dépenses contribuant à créer un actif possédant une valeur cessible sur le marché, il y a un pas que l'on ne saurait franchir au risque d'éliminer la totalité des actifs immatériels difficilement redéployables. Ce point fait l'objet d'un développement particulier dans la suite de l'article.

\subsection{Un niveau de risque élevé et atypique}

Les ETI de création récente présentent un niveau de risque élevé en relation avec la rapidité du développement qui les caractérise et le niveau d'incertitude induit par la dynamique innovatrice. De plus, la nature immatérielle d'une part significative de l'actif interdit toute prise de garantie gagée sur ce dernier et contribue à l'élévation du risque des apporteurs de capitaux.

\subsubsection{Un actif offrant une valeur de garantie problématique}

Dans les ETI, la valeur de l'actif repose essentiellement sur les possibilités de croissance et le capital humain. Le risque est donc élevé en cas de difficultés financières puisque alors, la valeur de ce type d'actif devient grandement problématique. Une illustration simple, se situant dans un contexte d'ouverture du capital, permet d'appréhender le risque rattaché à ce type d'entreprise. 
Vernimmen (1986) donne l'exemple fictif d'une société de haute technologie dont la valeur de marché s'élève à 40 millions de francs alors que le montant de ses capitaux propres comptables est de 1 million de francs. Si cette entreprise procède à une augmentation de capital d'un montant égal à sa valeur de marché, soit 40 millions de francs, les anciens actionnaires verront leur droit sur l'actif net être multiplié par 20,5. Faisons alors l'hypothèse que des difficultés intempestives et graves surviennent dès la fin de l'opération d'augmentation du capital, conduisant l'entreprise à envisager une dissolution se traduisant par une liquidation de l'actif. Les anciens actionnaires auront alors droit à 20,5 millions de francs, montant identique au droit des nouveaux actionnaires qui ont cependant apporté 40 millions de francs. La perte subie par ces derniers (égale dans notre exemple à 19,5 millions de francs) donne la mesure du risque inhérent à ce type d'entreprise et induit par la grandeur de la part de l'actif incorporel dans la détermination de la valeur de l'entreprise.

Par ailleurs, dans les situations de difficultés financières, le risque est grand de voir les employés, constitutifs de la valeur de l'actif technologique, quitter l'entreprise. Ce risque grandit à mesure que s'élève le risque de défaillance rendant d'autant problématique la valeur de cette composante de l'actif immatériel. De surcroît, la volonté de poursuite de l'activité dans un tel contexte se traduira probablement par l'obligation de consentir des garanties spéciales aux principaux clients pour les convaincre à maintenir leurs commandes à l'avenir (Brealey, Myers et Laroche, 1991). C'est dans un tel contexte qu'on perçoit que la confiance a un prix, et le capital de confiance, une valeur!

Tous ces éléments constituent autant de facteurs qui contribuent à la fragilisation des ETI de création récente et permettent d'affirmer qu'on est en présence d'entreprises à haut risque dans lesquelles les coûts de défaillance sont particulièrement élevés. On comprend dès lors l'attitude réservée des bailleurs de fonds traditionnels, et en particulier des prêteurs, généralement peu enclins à participer au financement de l'effort d'investissement de ces entreprises. Leur réticence est par ailleurs renforcée par la crainte qu'ils éprouvent de devoir participer, dans l'hypothèse d'un dérapage financier, fréquent dans ce type d'entreprise, dans le refinancement nécessaire à la restructuration financière de l'entreprise.

\subsubsection{L'évolution du risque au gré des phases de développement}

La relation entre la croissance, d'une part, et les turbulences et dysfonctionnements qui en résultent, d'autre part, a fait l'objet de nombreuses études. Il n'est donc pas nécessaire de développer cet aspect. Il en va de même de l'incertitude induite par le risque technologique de dépassement et d'obsolescence à la suite de la mise au point d'une innovation concurrente. Notre préoccupation présente est autre et se limitera à l'aspect financier. 
Nous avons évoqué le décalage qui sépare la création de l'actif technologique (phase de recherche-développement) de sa valorisation sur le marché (phases de démarrage puis de croissance). Cette dimension est fondamentale dans l'analyse de la problématique du financement des ETI, car elle met en évidence la spécificité des besoins de financement en relation avec l'évolution du risque rattaché à chacun d'eux.

Le premier point à prendre en compte est la forte évolution du taux d'échec en fonction des phases de la dynamique innovatrice. Des diverses études réalisées (Mansfield et Wagner, 1975; Urban et Hauser, 1980), on sait que seulement $20 \%$ des projets étudiés en phase de recherche atteindront la phase de développement, et que $60 \%$ seront abandonnés à ce stade. Les $40 \%$ qui franchissent l'obstacle subiront au niveau du lancement un taux d'échec qui s'étage de 30 à $50 \%$. Le taux de réussite final est donc faible: moins de 5\%; il illustre la forte incertitude qui caractérise l'évolution des ETI, avec les conséquences évidentes de ce phénomène sur leur financement.

Élément de complexité supplémentaire, l'incertitude technique et commerciale que nous venons d'évoquer se trouve renforcée par l'incertitude qui plane sur la stratégie des concurrents dont les réactions défensives peuvent avoir de fortes répercussions sur l'équilibre financier des ETI. En effet, l'adoption par la concurrence de pratiques de prix prédateurs aura un effet dépressif sur l'autofinancement qui ne pourra que contribuer à alimenter la réticence des bailleurs de fonds, notamment des banques.

Wedig (1990) a mesuré l'incidence du risque technologique sur le coût du capital mobilisé pour financer la création de l'actif technologique. Ce coût varie fortement selon le niveau de l'intensité concurrentielle et la taille de l'entreprise. En retenant les deux cas extrêmes, d'un côté l'ETI de grande taille évoluant dans une industrie faiblement concurrentielle, de l'autre l'ETI de petite dimension présente sur un marché fortement concurrentiel, Wedig suggère qu'on accorde une prime de risque de 0,5 à $1 \%$ dans le premier cas contre 5 à $9 \%$ dans le deuxième. L'amplitude est forte et révélatrice du haut niveau de risque qui peut être atteint dans les ETI.

\subsection{Des besoins de capitaux élevés}

Contraintes d'assumer des dépenses d'investissement élevées, majoritairement de nature immatérielle, les ETI ont la nécessité de mobiliser un volant de capitaux dont le montant est significativement plus élevé que celui des entreprises traditionnelles de même taille. Cela se traduit par un recours fréquent au capital de risque et un accès privilégié aux aides et financements publics qui donnent une configuration particulière à la structure financière de ces entreprises. 
Les industries et les entreprises technologiques constituent le secteur de prédilection du capital de risque (Geoffron, 1991). Le développement de l'industrie du capital de risque aux États-Unis illustre l'étendue des besoins de fonds propres des ETI. Les périodes de forte intensité d'investissement par le capital de risque dans un secteur donné précèdent généralement le décollage du secteur. Ainsi, le capital de risque a fortement été investi dans le secteur des semi-conducteurs de 1965 à 1971, année à partir de laquelle les ventes ont connu une croissance exponentielle.

En France, où l'industrie du capital de risque est moins développée, c'est l'État qui, à travers un système d'aides et de financements publics complexes, se substitue et satisfait en partie la demande de capitaux des ETI. Ainsi, les industries de haute technologie s'adjugent-elles $95 \%$ du financement public alloué à la recherche (Lehoucq et Strauss, 1988) et la moitié des entreprises de haute technologie perçoivent une aide publique à leur création (Albert et Mougenot, 1988).

Plusieurs études ont été réalisées qui permettent d'apprécier l'importance quantitative des besoins en capitaux des ETI de création récente et l'origine de l'offre de capital qui les couvre.

Il en ressort plusieurs constats:

- 1'apport de capitaux propres des créateurs est généralement complété par un apport en provenance d'investisseurs locaux souvent d'origine industrielle, et pour le solde, par des sociétés de capital de risque. Sur un échantillon de cent cas de créations d'entreprises innovantes de la région lyonnaise, ces capitaux propres «externes» représentaient $40 \%$ du total des capitaux propres (Albert et Mougenot, 1988b). Cette diversification de l'actionnariat constitue une caractéristique spécifique de la structure financière des ETI qui n'est bien évidemment pas sans avoir des retentissements organisationnels sur la vie de l'entreprise;

- les capitaux propres et les aides publiques représentent une part significative des capitaux mobilisés pour le financement des besoins des ETI. Une étude réalisée par Sofaris en 1986 montrait que les capitaux d'emprunt étaient limités au tiers des ressources stables, le solde étant financé aux deux tiers par des capitaux propres et pour le tiers restant, par des aides publiques (Sofaris, 1986). L'étude qui portait sur des entreprises de haute technologie à besoins élevés de capitaux n'en était que plus éclairante quant au rôle prépondérant et déterminant que jouent les capitaux propres dans le développement des ETI, contribution d'autant plus déterminante, semble-t-il, que l'intensité technologique est forte. 


\section{L'éclairage théorique du comportement financier des ETI}

À l'exception du rôle d'intermédiation des sociétés de capital de risque, force est d'admettre que le financement des ETI n'a pas constitué, jusqu'à des années récentes, un centre de préoccupation de la finance d'entreprise. Nous en avons pour preuve le nombre de publications consacrées à l'analyse économique de l'innovation.

Les progrès réels dans la compréhension du comportement financier de ces entreprises nous paraissent principalement découler des travaux de Williamson portant sur l'adéquation des modes de financement au degré de spécificité des actifs. Cependant, la théorie financière n'est pas en reste et fournit malgré tout un cadre conceptuel pertinent pour expliquer le comportement financier des $\mathrm{ETI}^{3}$. Reste à préciser la notion de spécificité appliquée à l'actif en vue de pouvoir mieux l'utiliser pour caractériser les diverses composantes de l'actif immatériel.

\subsection{Points de repère théoriques relatifs au financement de l'actif immatériel}

La nature et la composition de l'actif ne sont pas sans influencer la structure du capital de l'entreprise. Une caractéristique de l'actif, la spécificité de ses composantes, semble avoir un rôle déterminant dans le choix des modes de financement.

\subsubsection{Mise en évidence d'une corrélation négative entre l'importance de l'actif immatériel et le recours à la dette}

L'observation des structures financières des entreprises possédant des éléments d'actifs immatériels significatifs montrent que ces dernières ont tendance à moins recourir à la dette que les entreprises dont l'actif est principalement composé d'éléments matériels.

Ainsi, à partir d'un échantillon de 500 entreprises américaines, Long et Malitz (1985) ont mis en évidence une corrélation négative significative entre l'intensité des dépenses consacrées à la recherche-développement et à la publicité et le niveau d'endettement. De même, et constat corollaire de l'observation précédente, ces chercheurs ont trouvé une relation positive entre l'intensité de l'effort d'investissement matériel et le niveau d'endettement.

3. On trouve un panorama assez complet des apports de la théorie financière dans les documents de recherche de Adam et Farber $(1987$; 1988 ; 1994). On regrettera cependant l'absence de prise en compte des travaux de Williamson. 
Williamson (1985) avait précédemment fait les mêmes constats en suivant une démarche différente qui l'avait conduit à s'intéresser au décalage qui sépare la valeur de marché des capitaux propres de leur valeur patrimoniale, cette dernière prenant en compte le coût de remplacement (ou de reconstitution) de l'actif immatériel. Williamson avait montré que plus le décalage était grand, moins le niveau d'endettement était élevé.

Comme le souligne Myers et Maljuf (1984), il y a de nombreuses indications qui montrent que le niveau d'endettement n'est pas uniquement déterminé par la valeur et le risque de l'actif, mais aussi par la nature des éléments d'actif détenus par l'entreprise. Pour l'affirmer, il s'appuie sur l'idée que les entreprises ayant une valeur de marché des capitaux propres sensiblement plus élevée que leur valeur comptable devraient avoir, toutes choses égales par ailleurs, un ratio d'endettement élevé quand on exprime ce dernier par rapport à la valeur comptable des capitaux propres. L'observation infirme cette hypothèse et confirme le faible goût pour l'endettement des entreprises possédant une forte part d'actif immatériel, dont le décalage entre la valeur de marché des capitaux propres et leur valeur comptable est le reflet.

La hiérarchie des financements observés ou prescrits à partir de la théorie amène à se demander si la structure financière adoptée ne constitue pas une réponse au souci qu'ont les entreprises «immatérielles» de maintenir et de sauvegarder le capital de confiance qu'elles ont su créer auprès des nombreux agents avec lesquels elles sont en relation (clients, fournisseurs, bailleurs de fonds, État, personnel, collectivités locales, etc.). Comme le souligne Bloy (1994), la matière même de nombreux actifs immatériels est constituée par la confiance: "c'est par le long apprentissage de la confiance réciproque que s'établit le pouvoir de la marque, la fidélité de la clientèle, la reconnaissance du savoir-faire. Ces liens ne sont donc rien que de la confiance accumulée au cours du temps.»

Dans cette perspective, l'adoption d'un niveau d'endettement élevé qui contribue directement à accroître le risque de défaillance financière s'oppose à l'intérêt des agents économiques en relation avec l'entreprise (Cornell et Shapiro, 1987). Au premier rang desquels il conviendrait de placer, dans une ETI spécialisée dans la fabrication de biens d'équipements, les clients sécurisés par le contrat de garantie et de maintenance que l'entreprise est en mesure de leur offrir. Ce faisant, on s'oriente, comme le souligne Charreaux (1993), «vers une conception entièrement nouvelle du bilan de la firme [...] où la notion de capital organisationnel, définie comme la valeur de marché de l'ensemble des créances implicites futures que la firme projette de vendre, tient une place centrale». 
Cette question a fait l'objet de recherches originales de la part des économistes relevant du courant de l'organisation industrielle. Richardson (1978) a ainsi insisté sur l'ambivalence de la relation commerciale qui juxtapose ou superpose transaction de marché et action de coopération. Dans cette perspective, l'objectif de consolider la relation commerciale ne saurait alors s'accommoder d'une politique financière conduisant à une élévation du risque de défaillance en relation avec l'élévation de l'endettement.

\subsubsection{Rôle du degré de spécificité de l'actif dans le recours aux capitaux propres}

Par rapport aux enseignements traditionnels de la finance d'entreprise, Williamson (1988) introduit une novation en posant l'idée que la décision de financement est une transaction particulière où le degré de spécificité des actifs joue un rôle fondamental ${ }^{4}$. La spécificité d'un actif est définie par son aptitude à être interchangeable ou transférable, par sa faculté à être utilisé dans d'autres fonctions ou par d'autres utilisateurs (Williamson, 1989).

L'approche de Williamson conduit à appréhender l'entreprise comme une structure de gestion dans laquelle le comportement des agents économiques est régi par le principe de rationalité limitée et sujet à l'opportunisme. L'objectif est alors de restreindre le champ d'application de la rationalité limitée génératrice de contrats incomplets (contrats dans lesquels on ne peut préciser les états futurs du monde) et simultanément de protéger contre le risque de hasard moral induit par l'opportunisme des acteurs (Williamson, 1985).

Plutôt que de considérer les dettes et les capitaux propres simplement comme des modes de financement, Williamson propose de les analyser comme des structures de régulation des coûts de transaction générés par la nature de l'investissement. Dans cette perspective, le financement d'un actif devient une transaction dont il faut gérer le coût. La source principale des coûts de transaction réside alors dans les coûts d'adaptation qu'il faudrait éventuellement engager pour procéder aux ajustements ex post nécessités par l'inadéquation de la structure financière aux caractéristiques des éléments d'actif. Williamson propose d'analyser simultanément les caractéristiques des transactions et celles (notamment les coûts) des structures de régulation qui peuvent leur être associées sachant que la spécificité des éléments d'actif ressort comme la caractéristique la plus discriminante pour les différencier.

4. En cela, Williamson (1985) ne fait qu'étendre au domaine financier les principes de l'économie des coûts de transaction qui soutient que la spécificité de l'actif est la dimension essentielle pour caractériser les transactions. 
La démonstration de Williamson repose sur la mise en évidence du degré de spécificité comme facteur d'élévation du coût de la dette. En effet, l'élévation du degré de spécificité de l'actif, en risquant d'en interdire le financement par emprunt, peut conduire à l'abandon de projets d'investissements rentables. La mise en évidence de ce coût d'opportunité entraîne le recours à un autre mode de financement et justifie l'existence des capitaux propres.

Dans la logique du paradigme de la théorie des coûts de transaction, Williamson assimile la dette à un mode de gestion par le marché. La dette est une structure de régulation dont la seule obligation qui lui est rattachée est le respect des échéances. Si le débiteur se trouve dans l'impossibilité d'en supporter les charges, le prêteur cherchera à se dédommager en actionnant les garanties prises à son profit sur les éléments d'actif financés, garanties dont la valeur sera fortement dépendante du caractère redéployable des éléments en question.

Conséquence de ce phénomène, les termes du financement par la dette seront ajustés ex ante. Le prêteur dont l'exigence se fera de plus en plus forte à mesure que s'élèvera la spécificité des éléments d'actif, adaptera son comportement jusqu'au refus de financer. Le recours aux capitaux propres, qui présente la caractéristique d'être un financement accordé pour toute la durée de vie de l'entreprise, permet de surmonter ce risque. En contrepartie, l'entreprise se crée un certain nombre d'obligations, au premier rang desquelles figure le droit de regard des actionnaires, droit de regard qui s'étend précisément aux décisions d'investissement. En revanche, l'appel aux capitaux propres procure une certaine flexibilité dans la gestion des périodes de crise. En effet, l'actionnaire bénéficiant d'un statut de «créancier» résiduel sur les résultats et les éléments d'actif en cas de liquidation de ceux-ci est, de ce fait, peu sensible à la liquidité des éléments d'actif et donc à leur spécificité. C'est pourquoi, dans les situations de crise, l'actionnaire soucieux de préserver son patrimoine, est disposé, à la différence des prêteurs, à étudier les formules d'adaptation nécessaires à la pérennité de l'entreprise et ne remettant pas en cause l'outil économique dont elle s'est dotée.

Si on appelle $D(k)$ et $E(k)$, les coûts respectifs de la dette et des capitaux propres, $k$ étant le degré de spécificité des éléments d'actif, [ $k^{*}$, valeur de l'indice de spécificité pour lequel $D(k)=E(k)$ ] Williamson pose:

$$
\begin{aligned}
& \mathrm{D}(0)<\mathrm{E}(0) \\
& D^{\prime}(k)>E^{\prime}(k)>0
\end{aligned}
$$




\section{Coût du financement et spécificités des éléments d'actif}

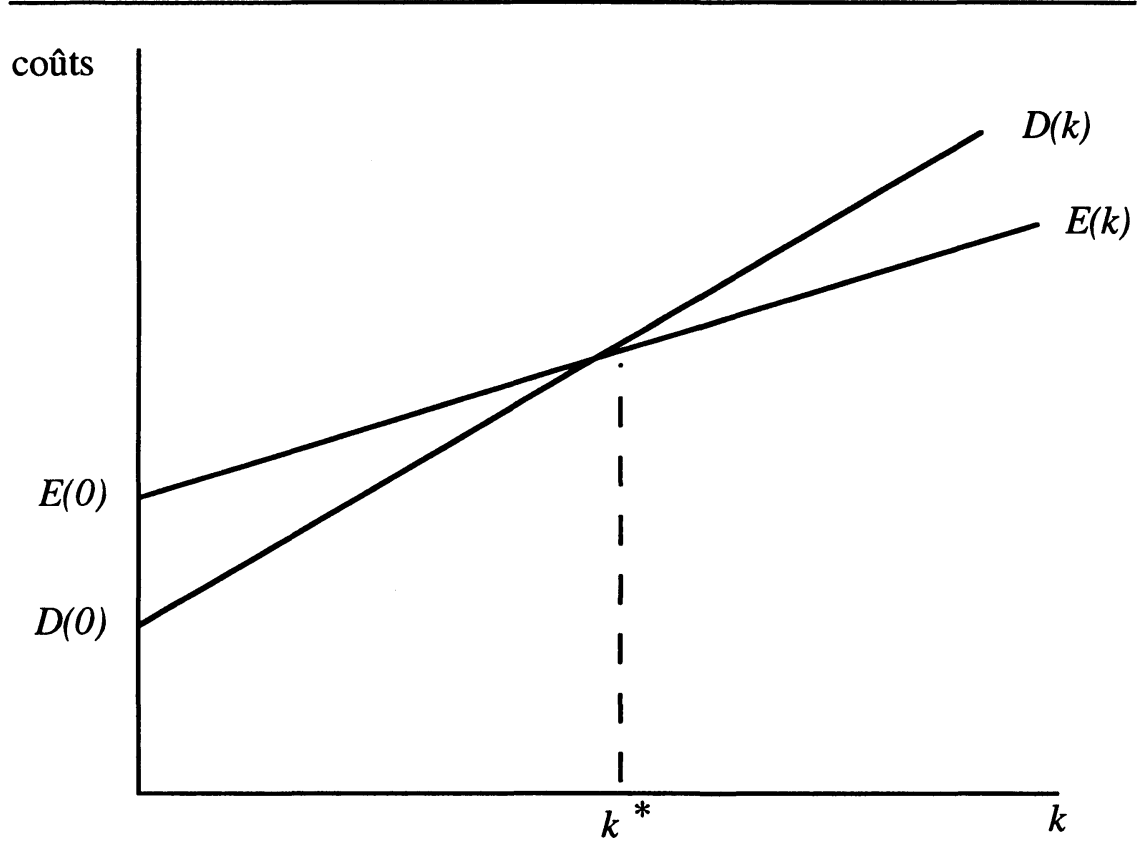

* La première inégalité traduit l'existence de coûts fixes au regard des capitaux propres plus élevés que ceux de la dette. À la différence de la dette qui est une structure de régulation simple régie par des règles définies ex ante, le financement par capitaux propres induit des relations de gestion complexes, nécessitant notamment une implication des apporteurs de capitaux. Ces aménagements d'ordre privé, totalement indépendants de la spécificité des éléments d'actif, engendrent les coûts fixes.

* La deuxième inégalité montre que les coûts de la dette augmentent plus rapidement que ceux des capitaux propres en relation avec l'élévation du risque de liquidation et l'apparition d'un risque d'abandon de projets créateurs de valeur. Par comparaison, le coût des fonds propres est peu sensible à la spécificité des éléments d'actif.

La minimisation des coûts de transaction engendrés par la nature de l'investissement est alors obtenue en associant la dette aux projets d'investissements redéployables $\left(k<k^{*}\right)$ et en finançant par capitaux propres les éléments d'actif spécifiques $\left(k>k^{*}\right)$. Ce faisant, on associe une structure de régulation régie par des règles prédéterminées (la dette) aux actifs présentant une faculté de réalisation (actifs redéployables). A contrario, on associe une structure de régulation offrant une liberté d'action certaine (les capitaux propres) au financement d'actifs non redéployables, ce que Williamson (1988) exprime en une formule condensée: "l'adoption de structures de régulation complexes et coûteuses qui remplacent les règles par la liberté d'action peut être, et est souvent, la source de création de valeur lorsque l'incertitude se développe». 
De cette proposition découle un certain nombre de conséquences.

- La dette et les fonds propres sont des structures de gestion des coûts de transaction engendrés par la nature de l'investissement entre l'entreprise, ses prêteurs et ses actionnaires. Le mode de financement adopté pour un investissement donné relève de la volonté de gérer les coûts de transaction induits conjointement par la spécificité des éléments d'actif et les comportements des acteurs (rationalité limitée, opportunisme). Lorsque l'entreprise recourt au marché, c'est-à-dire à la dette, pour financer ses investissements, il y a une transaction visant à rétablir l'optimalité du prix du service. Le coût de la transaction est d'autant plus élevé que l'actif financier est peu redéployable. À l'inverse, lorsque l'entreprise fait appel aux actionnaires, il y a économie des coûts de transaction induits par la spécificité des éléments d'actif.

- C'est à ce niveau que l'analyse peut être utilement complétée par la prise en compte des coûts d'agence entraînés par un recours aux capitaux propres. Il devient alors pertinent d'intégrer l'éclairage de la théorie de l'agence qui voit dans la dette et les capitaux propres des modes de financement permettant de gérer l'asymétrie d'information inhérente aux relations entre l'entreprise, d'une part, ses créanciers et actionnaires, d'autre part.

- Le principe de séparabilité des décisions d'investissement et de financement vole en éclat. Le coût de la dette et la nature des investissements sont interdépendants et doivent être pris en compte simultanément (Williamson, 1989). Le coût de transaction n'est pas engendré par la dette, mais dû au caractère redéployable de l'actif financé. Il y a une remise en cause de l'approche regroupements de fonds développée par la finance moderne. La notion de structure financière optimale disparaît au profit d'une analyse de la décision de financement de l'actif qui rejoint les préoccupations de la théorie du financement hiérarchique (Pecking Order Theory).

- Dans cette perspective, il convient cependant de s'interroger sur le caractère interactif des décisions financières, en se demandant notamment si la spécificité des éléments d'actif est la variable déterminante dans la mesure où elle est, elle-même, le reflet de choix stratégiques qui dictent la configuration de l'actif économique mis en œuvre dans le processus d'exploitation (Charreaux, 1993).

- Cette interrogation paraît confortée par le développement récent, au sein du courant de l'organisation industrielle, de réflexions sur le sens des relations de causalité pouvant être établies entre le degré de spécificité des éléments d'actif et le choix des structures organisa- 
tionnelles. En d'autres termes, la spécificité des éléments d'actif estelle une donnée exogène dont découle le choix d'un type de structure financière ou organisationnelle, ou au contraire, est-elle la résultante de choix stratégiques, organisationnels, voire financiers?

- Transposée dans la problénatique du financement de l'ETI, l'approche de Williamson débouche sur un message clair et sans ambiguïté. Au regard de l'importance de l'actif immatériel et du risque, dont la conjonction contribue à un degré de spécificité de l'actif particulièrement élevé, le financement à adosser à la stratégie de développement des ETI ne peut être qu'un recours massif aux capitaux propres accompagné d'un contrôle étroit des actionnaires.

\subsection{Enseignements positifs pour la stratégie financière des ETI}

Les ETI recourent fortement à l'autofinancement et aux capitaux propres. La logique de cette séquence de financement est justifiée par certains éclairages théoriques. De là à penser que la stratégie financière des ETI dispose d'un cadre normatif opératoire, il y a un seuil qui ne saurait être franchi qu'après avoir éclairci les ramifications et les distinctions qui existent entre la spécificité et le caractère immatériel des éléments d'actif.

\subsubsection{Justification de la séquence de financement spécifique}

Interrogés à l'occasion d'une enquête réalisée sur le financement des investissements immatériels, les dirigeants de PMI exprimaient une préférence marquée pour le financement par ressources propres en général, et pour l'autofinancement en particulier (Sofaris, 1988). Cette séquence de financement diffère de la séquence traditionnellement observée, laquelle place le recours à l'emprunt au deuxième rang après l'autofinancement, mais avant l'appel aux capitaux propres.

Au regard des considérations théoriques précédemment développées, la hiérarchie de financement exprimée par ces dirigeants répond à une logique économique certaine. En effet, la majorité des éléments d'actif immatériels présente un degré de spécificité élevé qui interdit d'envisager, en cas de liquidation, leur réaffectation vers un autre usage. Leur financement par ressources propres (autofinancement ou capitaux propres) est donc une réponse adaptée aux caractéristiques des investissements entrepris.

Ce constat conduit aussi à rejeter les critiques qui sont parfois exprimées à l'égard des banques à qui il est reproché une certaine hésitation pour financer par de la dette l'effort d'investissements immatériels. Cette réticence a un 
fondement économique qu'il convient d'intégrer avant de dénoncer une quelconque frilosité dans leur attitude!

La préférence pour l'autofinancement a fait l'objet d'attention de la part de la théorie financière et a donné lieu à une formulation que nous allons brièvement présenter. Nous compléterons cet éclairage en rappelant les principaux enseignements de la théorie financière traditionnelle (théorie de l'arbitrage statique) qui fournit, elle aussi, un cadre explicatif du comportement financier des ETI.

\section{La théorie de l'ordre de préférence}

\section{dans le choix des sources de financement}

La théorie d'une hiérarchie des financements est déjà ancienne puisque sa première formulation a été présentée par Donaldson (1981) qui, déjà en 1961, soulignait la préférence des dirigeants pour un recours à l'autofinancement. Ce constat bouleversait nombre d'enseignements de la finance traditionnelle en mettant en évidence un comportement « déviant » (la préférence pour l'autofinancement) qui pouvait apparaître comme un moyen saisi par les dirigeants pour s'abstraire de la logique et de la contrainte du marché financier. Par la suite, cette théorie a fait l'objet d'un renouvellement complet à l'initiative de Myers et Majluf (1984) qui ont trouvé dans la théorie de l'asymétrie d'information les concepts explicatifs de ce comportement financier.

Dans un contexte d'asymétrie informationnelle (les dirigeants ont connaissance de la vraie valeur des projets), Myers et Majluf démontrent que si l'objectif des dirigeants est la maximisation de la richesse des actionnaires actuels, les dirigeants chercheront à les protéger contre le risque potentiel de sous-évaluation, générateur d'un transfert de richesse au profit des nouveaux actionnaires, inhérent à l'émission de nouvelles actions. Les investisseurs prenant en compte ce risque, l'interaction finale des comportements des acteurs peut conduire à des situations de sous-optimalité. Ainsi, les dirigeants préféreront ne pas émettre d'actions nouvelles, car cela reviendrait à signaler que les conditions de l'opération sont défavorables pour les nouveaux actionnaires. Conséquence paradoxale de la situation : les dirigeants vont renoncer à un projet d'investissement créateur de valeur $(V A N>0)$ si le financement du projet doit être assuré par l'émission de nouvelles actions.

Dans ces conditions, on comprend aisément les raisons qui incitent les dirigeants à privilégier l'autofinancement au détriment du financement externe. À la suite des propositions de Myers et Majluf, la nature des coûts liés à un financement par augmentation de capital (coûts administratifs, coût de sous-évaluation) doit être étendue à la prise en compte du 
coût d'opportunité correspondant au manque à gagner assumé par les actionnaires dans l'hypothèse de l'abandon d'un projet créateur de valeur. Rappelons que cet abandon est motivé par la crainte de procéder à une émission d'actions sous-évaluées. Ce coût disparaît si l'entreprise a suffisamment d'autofinancement pour réaliser les projets dont la VAN est positive. La préférence des dirigeants pour l'autofinancement apparaît alors comme une tentative d'éviter ce coût d'opportunité en donnant la possibilité à l'entreprise de pouvoir investir sans devoir recourir à une augmentation de capital.

Ce n'est que lorsque l'autofinancement s'avère insuffisant que l'entreprise fait appel au financement externe, mais en privilégiant la dette par rapport à l'émission d'actions nouvelles. La préférence alors manifestée pour la dette est la conséquence de l'asymétrie d'information entre dirigeants et nouvel actionnaire, et du risque, pour ce dernier, que l'entreprise n'émette des actions que si les titres doivent être surévalués. Dans ces conditions, un nouvel actionnaire ne souscrira à une augmentation de capital que lorsque l'entreprise aura préalablement utilisé son potentiel d'endettement. Est ainsi légitimée la séquence de financement conduisant à recourir dans un premier temps à l'autofinancement, puis à la dette et, enfin, à l'émission d'actions.

\section{La théorie de l'arbitrage statique}

Les coûts de faillite sont le facteur le plus souvent évoqué pour expliquer le fait observé que les entreprises ne s'endettent pas à l'infini. En les prenant en compte, la théorie de l'arbitrage complète le modèle développé par Modigliani et Miller. La théorie de l'arbitrage (Static Trade Off Theory) définit la structure financière optimale comme le résultat d'un processus d'arbitrage dont découle le coût du capital minimal permettant de maximiser la valeur de l'entreprise. Le coût du capital est notamment déterminé par la différence entre les coûts de défaillance et les économies d'impôt liées à l'endettement. Toute élévation de l'endettement, en même temps qu'elle procure un surplus d'économie d'impôt, contribue à une augmentation des coûts de faillite. D'où la nécessité de devoir arbitrer des intérêts contraires.

\section{La hiérarchie de financement spécifique aux ETI}

Dans le prolongement de la théorie de l'arbitrage, deux raisons peuvent être avancées, permettant de comprendre la place réduite accordée à la dette dans le financement des ETI :

- la nature des éléments d'actif possédés, dont le degré de spécificité est particulièrement marqué, induit un coût de la dette élevé, 
entraîné par l'élévation du coût de faillite en relation avec l'importance des coûts de liquidation de l'actif;

- l'avantage représenté par la déductibilité des frais financiers de la base d'imposition est problématique dans les ETI dans la mesure où le niveau de résultat, grevé par la comptabilisation en charge de l'essentiel de l'effort d'investissement immatériel, ne permet que rarement de profiter pleinement de l'économie d'impôt.

De la sorte, on comprend que l'endettement, privé de son avantage fiscal, handicapé par l'importance des coûts de faillite inhérents aux coûts de liquidation de l'actif incorporel, soit le parent pauvre au sein des concours mis en place pour assurer le financement du développement des ETI.

Cependant, les raisons précédentes paraissent insuffisamment explicatives. On observe dans la réalité que les entreprises performantes, dégageant des résultats élevés, s'endettent peu (Brealey, Myers et Laroche, 1991), se privant volontairement du bénéfice de l'avantage fiscal. Ce constat conduit dès lors à se demander si le faible endettement des ETI n'est que la contrepartie de la spécificité de l'actif détenu ou s'il ne traduit pas aussi la capacité de ces entreprises à dégager des résultats suffisants pour permettre un autofinancement significatif de leur effort d'investissement. La question mérite d'être posée au regard de l'importance de l'autofinancement des dépenses de recherche ${ }^{5}$.

Ce faisant, on rejoint l'éclairage de la théorie des préférences qui montre que l'arbitrage entre les avantages fiscaux et les coûts de faillite ne joue que sur le besoin de financement résiduel, après affectation prioritaire de l'autofinancement à l'effort d'investissement. Lorsqu'un besoin de financement subsiste, mais alors seulement, les entreprises suivent la séquence de financement emprunt-capitaux propres, utilisant en priorité les financements les moins risqués. Dans les ETI, au regard du poids de l'actif immatériel, on passera directement de l'autofinancement au financement externe par capitaux propres, le recours à l'endettement n'étant utilisé que pour le financement résiduel de l'actif matériel.

Les deux théories explicatives du comportement de financement des entreprises nous livrent des enseignements plus complémentaires qu'opposés qui fournissent un cadre explicatif cohérent du comportement financier des ETI. Sont notamment justifiées la nécessité et l'importance du recours aux capitaux propres et, plus particulièrement, au capital de risque. De surcroît, ces enseignements permettent d'avancer une hypothèse: que les résultats dégagés

5. En France, le taux d'autofinancement des dépenses de recherche et développement s'élève à $78 \%$ selon les résultats d'une récente enquête du ministère de l'Industrie (1993). 
par ces entreprises sont sûrement plus élevés qu'on le dit habituellement, et qu'on n'a pas, sur le plan comptable, la possibilité de l'apprécier6.

\subsubsection{De la nécessité de mieux caractériser la spécificité de l'actif immatériel}

Après avoir noté que l'élévation du degré de spécificité des éléments d'actif est un phénomène qui paraît s'être accentué au cours des dernières années, nous préciserons la notion de spécificité en nous efforçant de distinguer entre actif spécifique et actif immatériel.

\section{La tendance à l'élévation de la spécificité des éléments d'actifs}

Comme le souligne Everaere (1993), «la spécificité signifie des caractéristiques singulières, donc limitées ou peu répandues [...]». C'est pourquoi la perte de valeur en cas de réutilisation est souvent citée comme une des caractéristiques essentielles d'un actif spécifique. Analysée à la lumière de cette caractéristique, l'évolution technique et technologique des dernières décennies paraît s'être accompagnée d'une élévation du degré de spécificité des différents éléments d'actif mis en œuvre dans les processus productifs.

L'observation de la vie des affaires fournit de nombreux exemples d'actifs corporels ayant subitement perdu toute valeur d'utilisation par suite de modifications profondes du contexte dans lequel ils étaient employés. Ce phénomène est renforcé par la recherche de la différenciation dans la conception, la fabrication et la distribution des produits. L'adaptation des processus de production et des circuits de distribution qui en découle connaît alors une évolution opposée à la banalisation et induit naturellement un renforcement de la spécificité des éléments d'actif.

Il est facile d'illustrer le propos en prenant l'exemple de l'industrie du luxe dans laquelle l'adoption naturelle d'une stratégie de différenciation nécessite généralement que l'entreprise acquière et mobilise des ressources spécifiques. Est là pour le montrer l'exemple de Hermès qui, pour garantir la qualité de ses produits, a fait le choix de se doter de sa propre «école du cuir» et a procédé au rachat de plusieurs fournisseurs (Moingeon et Kikuno, 1994).

La part croissante du rôle de l'information dans l'économie peut aussi s'analyser comme un souci de mise en valeur des caractéristiques

6. Ces entreprises optent rarement pour une immobilisation de leur actif immatériel, option coûteuse fiscalement, préférant autofinancer le maximum de leurs investissements immatériels en les comptabilisant dans les charges d'exploitation. 
distinctives des produits en cherchant à accroître les connaissances du consommateur sur les produits. La part des ressources allouées aux activités de promotion commerciale n'a fait que croître au cours des dernières années, contribuant à la création d'un actif immatériel spécifique à un produit ou à un portefeuille de produits.

\section{Distinguer immatérialité et spécificité des éléments d'actif}

L'assimilation est souvent faite entre actif immatériel et actif spécifique. S'il apparaît, à l'examen, que la majorité des éléments d'actif immatériels présente un degré de spécificité élevé, ce constat ne doit cependant pas conduire à confondre les deux notions.

Tous les éléments d'actif immatériels ne sont pas spécifiques et inversement. Certains éléments physiques présentent une redéployabilité réduite alors que d'autres, immatériels, méritent d'être rattachés à la catégorie des éléments d'actif redéployables dans la mesure où leur valeur ne sera pas altérée en cas de revente.

Pour éclairer le débat, on peut s'appuyer sur une taxonomie des éléments d'actif immatériels et rapprocher les catégories ainsi définies de la typologie des spécifités propres à ces éléments suggérée par Williamson.

Dans un premier temps, Williamson (1989), a distingué cinq types de spécificité:

- la spécificité de site qui repose sur la localisation et l'agencement d'éléments d'actif physiques dont l'enchaînement et la complémentarité dans les procédés sont génératrices d'économies de coût ;

- la spécificité d'application qui caractérise les éléments d'actif physiques utilisés dans un processus productif particulier;

- la spécificité des éléments d'actif humains dont le fondement est constitué par les compétences et les savoir-faire mis en œuvre dans l'entreprise ;

- la spécificité des éléments d'actif dédiés à un débouché unique ;

- la spécificité de la notoriété et de la réputation acquise.

Par la suite, Williamson (1991) a introduit une catégorie supplémentaire en distinguant une spécificité temporelle qui traduit une situation de nonséparabilité technologique des éléments d'actif pouvant découler de la forte interdépendance des éléments d'actif humains et physiques sur un site d'exploitation. 
À ces types de spécificité se rattachent, selon les cas, des éléments d'actif physiques et (ou) immatériels. Rapprochons cette grille de lecture de la typologie des éléments d'actif incorporels proposée par Glais et Sage (1989).

Ces derniers distinguent deux grandes catégories: d'une part, les éléments incorporels assimilables à des éléments corporels; d'autre part, les éléments incorporels de nature immatérielle.

À la première catégorie se rattachent les «rentes» provenant de l'acquisition d'un droit (exemple: droit au bail, brevet) ou de l'exploitation d'une autorisation administrative (exemple: licence de transport). Les éléments incorporels présentent souvent un caractère durable qui ne peut cependant être étendu à l'ensemble de l'actif incorporel concerné. Ainsi, l'aléa rattaché aux revenus des brevets est évident et contredit la durabilité précédemment mise en avant.

Au regard des critères proposés par Williamson, la spécificité de ce groupe d'éléments d'actif paraît faible. Leur possibilité de négociation est réelle et fortement dépendante de leur transmissibilité. La redéployabilité de ces actifs découle de la durée et de l'importance de l'avantage transmis au nouvel acquéreur. Dans les ETI, cette catégorie d'actif immatériel occupe une place marginale.

La deuxième catégorie d'actif distingué par Glais et Sage réunit les éléments incorporels de nature immatérielle auxquels se rattachent l'actif technologique et l'actif commercial. L'actif technologique recouvre le stock de savoir-faire accumulé par l'entreprise; l'actif commercial traduit fondamentalement l'existence et la durabilité du lien établi entre l'entreprise et ses clients.

L'analyse, à la lumière de la typologie de Williamson, des éléments d'actif immatériels réunis dans cette deuxième catégorie montre qu'il s'agit pour l'essentiel d'éléments dont le degré de spécificité est élevé.

Deux raisons paraissent l'expliquer:

- la faible séparabilité de ces éléments d'actif par rapport aux autres fonctions et ressources de l'entreprise ;

- le fait que, comme le souligne Gaffard (1990), «les coûts supportés pour les mettre en œuvre en vue d'une certaine application ne sont pas recouvrables si les actifs en question sont redéployés vers d'autres applications ». Plus la part des coûts irrécouvrables est forte au sein des dépenses immatérielles engagées, plus la spécificité des éléments d'actif créés sera élevée. 
Cette deuxième catégorie d'actif, notamment l'actif technologique et l'actif commercial, occupent une place significative au sein de l'actif des ETI. On est là en présence d'éléments d'actif immatériels qui relèvent fondamentalement d'une logique de la relation, à la différence de la catégorie précédente, et pour lesquels la confiance des différents partenaires de l'entreprise constitue l'essence même de leur valeur (Bloy, 1994).

\section{Conclusion}

Des développements précédents, plusieurs idées ressortent.

L'ampleur de l'effort d'investissement induit par la dynamique du processus de développement technologique contribue à engendrer dans les ETI de création récente un besoin structurel de capital. Ce besoin, contrepartie de l'actif immatériel créé par l'engagement de dépenses spécifiques de nature technologique et commerciale, est couvert, dans le cadre d'une hiérarchie de financement spécifique, d'une manière préférentielle par l'autofinancement et, pour le solde, par un recours privilégié aux capitaux propres.

Dans ce cadre, la politique financière se voit assigner un objectif prioritaire: entretenir et sauvegarder l'actif technologique et commercial dont dépend le capital de réputation et sur lequel repose l'image technologique de l'entreprise et, donc, en dernier ressort, sa pérennité. La politique financière peut d'ailleurs aller au-delà de cet objectif et contribuer à la valorisation de l'actif. Tel nous paraît être le résultat à attendre de l'intervention d'un risqueur de capital dont l'entrée au capital constitue un effet de signal qui n'est pas sans influencer l'environnement de l'entreprise.

L'introduction du capital de confiance dans le champ de la politique financière des ETI pose ouvertement la question d'un élargissement des composantes de l'actif immatériel en direction de l'ensemble des partenaires de l'entreprise (clients, salariés, banque, etc.).

Cet élargissement paraît d'ailleurs d'autant plus s'imposer dans le cas des ETI au regard du rôle que jouent dans la constitution de l'actif organisationnel les contrats implicites passés entre l'entreprise et ses divers partenaires (Cornell et Shapiro, 1987). Se pose alors la question de la durabilité de l'effort de création du capital de réputation (Hall, 1992; Glais, 1983) et des conditions du maintien de la valeur de ce capital au premier rang desquels la politique financière occupe une place réservée.

Le dernier, mais non le moindre, la relation établie entre la constitution d'un actif immatériel et son financement mérite spécialement réflexion dans le cas des nouvelles ETI au regard de l'effet social du processus de développement de ce type d'entreprise. Le rôle économique des ETI déborde de la 
seule sphère marchande dans laquelle elles s'insèrent. On connaît désormais le rôle d'entraînement que le développement de ces entreprises peut avoir, comme modèle de réussite, sur l'aménagement du territoire (Miller et Coté, 1985). Raison de plus pour s'interroger sur la contribution de la politique financière à la protection de la valeur créée par les ETI.

\section{Bibliographie}

ACS, Z.J. et D.B. AUDRESTSCH (1988), «Innovation in large and small firms: an empirical analysis», American Economic Review, vol. 78, $\mathrm{n}^{\circ} 4$.

ADAM, M.C. et A. FARBER (1987), «Le financement de l'innovation technologique: les caractéristiques de l'investissement novateur», Cahiers économiques de Bruxelles, vol. 116, $4^{\mathrm{e}}$ trimestre, p. 3-23.

ADAM, M.C. et A. FARBER (1988), «Le financement de l'innovation technologique: l'apport de la théorie financière», Cahiers économiques de Bruxelles, vol. 117, $1^{\text {er }}$ trimestre, p. 3-36.

ADAM, M.C. et A. FARBER (1994), Le financement de l'innovation technologique, Paris, Presses universitaires de France.

ALBERT, P.H. et P.H. MOUGENOT (1988), «La création d'entreprise haute technologie», Revue Française de Gestion, mars, p. 114-116.

BLOY, E. (1994), «Les biens incorporels: une valorisation fondée sur la confiance», Conférence donnée dans le cadre du séminaire Nouvel Institutionnalisme, 24 février, Université Lyon II.

BREALEy, A., S.C. MYers et P. LAROCHE (1991), Principes de gestion financière des sociétés, $2^{\mathrm{e}}$ éd., Montréal, McGraw-Hill, chapitre 36, p. 698 et chapitre 18.

Charreaux, G. (1993), «Théorie financière et stratégie financière», Revue Française de Gestion, janvier-février, (a) p. 55; (b) p. 59.

CORNELL, B. et A. SHAPIRO (1987), «Corporate stakeholders and corporate finance», Financial Management, printemps, p. 5-14.

Donaldson, G. (1981), Corporate Debt Capacity, Harvard GSB.

EVERAERE, C.H. (1993), «Des coûts aux investissements de transaction: pour un renversement de la théorie de Williamson», Revue Française d'Economie, $\mathrm{n}^{0} 4$, p. 162.

FAU, I. (1993), «Le diagnostic économique des pépinières d'entreprises innovantes», Mémoire de DESS, IAE/ Université Lyon III. Les principaux résultats de cette étude ont été repris dans un rapport publié par la Chambre de commerce et d'industrie de Lyon, Novacité : étude d'impact économique, octobre.

GAFFARD, J.L. (1990), Économie industrielle et de l'innovation, Paris, Dalloz, p. 308. 
GEOFFRON, P. (1991), «Le financement des PME innovatrices: l'exemple américain », Revue Française de Gestion, juin, p. 131.

GLAIS, M. et E. SAGE (1989), «Évaluation des actifs incorporels», dans Encyclopédie de Gestion, Paris, Vibert, p. 1189.

GlaIS, M. (1993), Économie industrielle: les stratégies concurrentielles des firmes, Paris, Litec, p. 130.

GODENER, A. (1994), «Entreprises traditionnelles et entreprises de haute technologie, des chemins de croissance différents?», XII ${ }^{\mathrm{e}}$ Journées Nationales des IAE, CREGO, IAE Montpellier, vol. 2, p. 184.

HALL, R. (1992), «The strategic analysis of intangible resources», Strategic Management Journal, vol. 13, p. 141.

HICKS, J. (1975), Le temps et le capital, Paris, Economica, p. 177.

INSEE (1993), Rapport sur les comptes de la Nation, L'économie française en 1992.

LARRERA DE MOREL, B. (1992), «Financer la technologie», La lettre de Sofaris, $\mathrm{n}^{0} 14$, janvier, p. 1.

LARUE DE TOURNEMIRE, R. (1991), «La modélisation stratégique dans les industries fondées sur la science», Revue Française de Gestion, juin-juillet, p. 92.

LEHOUCQ, T.H. et J.P. STRAuSS (1988), «Les industries françaises de haute technologie», Économie et statistiques, n ${ }^{0} 207$, février (a) p.15; (b) p.19.

LONG, M. et I. MALITZ (1985), «The investment financing nexus: some empirical evidence», Midland Corporate Finance Journal, vol. 3, automne, p. 53-59.

MahieuX, X. (1993), "Capital-investissement et valeur de l'entreprise», Banque, $\mathrm{n}^{0} 538$, juin, p. 63-66.

MANSFIELD, E. et S. WAGNER (1975), «Organizational and strategic factors associated with probabilities of success in industrial research and development», Journal of Business, vol. 48-2, avril.

MAIRESSE, J. et P. MoHNEN (1990), «Recherche-développement et productivité : un survol de la littérature économétrique », Économie et statistiques, n ${ }^{\text {os }} 237-238$, novembre-décembre, p. 103.

MARION, A. (1987), «Problématique financière de l'investissement immatériel», Analyse Financière, $1^{\mathrm{er}}$ trimestre, p. 72-83.

MARION, A. (1988), «La place de l'investissement immatériel dans l'évaluation des entreprises », Revue Française de Gestion, janvier, p. 6-12.

MARION, A. (1990), «La valorisation directe de l'actif immatériel: points de repère», Banque, mars, p. 240 et s.

Miller, R. et M. COTÉ (1985), «Growing the next Silicon Valley », Harvard Business Review, juillet, p. 41. 
MinISTÈRE DE L'INDUSTRIE (1993), Science et Technologie, indicateur 1994, Paris, Economica.

MoINGEON, B. et D. KIKUNO (1994), «Innover, c'est conjuguer stratégie et mise en œuvre», L'Expansion Management Review, automne, p. 86.

MYERS, S.C. et N. MALJUF (1984), «Corporate financing and investment decisions when firms have information investors do not have», Journal of Financial Economics, vol. 13-2, juin, p. 187-221.

RICHARDSON, G.B. (1978), «The organisation of industry », Economic Journal, septembre.

SOFARIS (1986), «Innovation: le poids de la recherche et développement », La Lettre de Sofaris, $\mathrm{n}^{0} 7$, novembre.

SOFARIS (1988), «L'investissement en matière grise des $P M E »$, sondage réalisé par la Sofaris, Colloque du 24 février 1988, Sofaris, p. 4.

TEILlET, P. (1988), «La mesure du coût des investissements immatériels», INSEE, Collection Archives et Documents, p. 99.

Urban, G. et J. HaUSer (1980), Design and Marketing of New Products, Englewoods Cliffs, N.J., Prenctice-Hall.

VERNIMmEn, P. (1986), Finance d'entreprise: Logique et politique, $2^{\mathrm{e}}$ éd., Paris, Dalloz, p. 239.

WEDIG, G. (1990), «How risky is research and development? A financial approach », Review of Economic and Statistics, vol. 72-2, mai, p. 296-303.

WiLliamson, O.E. (1981), «The moral hazard theorie of corporate financial structure: an empirical test», Conférence doctorale, MIT. (Cité par Myers, 1984.)

Williamson, O.E. (1985), The Economic Institution of Capitalism, New York, The Free Press, p. 30-32.

WILliAMSON, O.E. (1988), «Corporate finance and corporate governance», Journal of Finance, vol. 43, p. 589.

Williamson, O.E. (1989), «Transaction cost economic», dans R. Schmalensee et R.D. Willig (éd.), Handbook of Industrial Organization, Amsterdam, Elsevier Science Publishers, vol. 1, p. 142-143, vol. 2, p. 168-169.

Williamson, O.E. (1991), «Comparative economic organization: the analysis of discrete structural alternatives», Administrative Science Quarterly, vol. 36, $n^{0} 2$, juin, p. 269-296. 